\section{Advantages of heat-stable vaccines}

SIR-I agree with Arya' that a desired criterion for new chimaeric poliovirus vaccines should be stability to warm temperatures. It should be pointed out, however, that live poliovirus vaccines that contain a thermal stabilizer have been available from several manufacturers and in use in many countries, including the United Kingdom, for more than 20 years.

In the early $1960 \mathrm{~s}$ it was found that the infectivity of the enteroviruses could be preserved even when they were heated at $50{ }^{\circ} \mathrm{C}$, if molar $\mathrm{MgCl}_{2}$ was added ${ }^{2}$, a property that is still used in their identification and characterization. This finding was quickly applied to the live vaccine ${ }^{3}$, not only in laboratory manipulations ${ }^{4}$ but also in the field, where stabilized vaccines, in the absence of a cold chain, were used effectively to halt type 1 and type 3 outbreaks in a semitropical country ${ }^{5}$.

In mass poliovirus immunization programmes, particulary in developing tropical countries where it is difficult to maintain or to transport the vaccine under frozen conditions, the stabilized vaccine offers numerous advantages ${ }^{6}$.

In laboratory studies with live poliovaccine stored in $\mathrm{MgCl}_{2}$, investigators at Wellcome Research Laboratories ${ }^{7}$ found that the vaccine showed so little loss in virus titre after long-term storage at $-20{ }^{\circ} \mathrm{C}$ that the predicted half-life was calculated as 92 years. They further found that $\mathrm{MgCl}_{2}$-stabilized vaccine suffered no significant loss of potency after as many as nine cycles of alternate warm and cold conditions. The samples were alternately exposed to ambient temperatures and to storage frozen at $-20^{\circ} \mathrm{C}$ or liquid at $7-9{ }^{\circ} \mathrm{C}$.

Although this handling certainly cannot be recommended as a general practice, it is reassuring to know that properly stabilized vaccine can survive some of the 'cold chain' problems that have arisen in field use. Similar results obtained by another manufacturer ${ }^{8}$ indicate that $\mathrm{MgCl}_{2}$-stabilized vaccine retains potency in field and shipping conditions without refrigeration for 2 weeks, including periods when the daytime temperature reached $42{ }^{\circ} \mathrm{C}$.

\footnotetext{
1. Arya, S.C. Nature 332, 789 (1988).

2. Wallis, C. \& Meinick, J.L. Virology 16, 504-505 (1962)

3. Melnick, J.L., Ashkenazi, A., Midulla, V.C., Wallis, C. \& Bernstein, A. J. Am. med. Ass. 185, 406-408 (1963).

4. Petersen, I. \& von Magnus, H. Acta Path. Microbiol. Scand 61, 652-653 (1964).

5. Yofe, J., Goldblum, N., Eylan, E. \& Melnick, J.L. Am. J. Hyg. 76, 225-238 (1962)

6. Mirchamsy, H., Shafyi, A., Mahinpour, M. \& Nazari, P. Vaccinations in Developing Countries: Dev. Biol. Standar diz. 41. 255-257 (Karger, Basel, 1978).

7. Finter, N. B., Ferris, R.. Kelly, A. \& Prydie, J. Vaccinations in Developing Countries: Dev. Biol. Standardiz. 41, 271-276 (Karger, Basel, 1978)

8. Peetermans, J., Colinet, G. \& Stephenne, J. Proc. Symp. Stability and Effectiveness of Measles, Poliomyelitis, and Pertussis Vaccines, 61-66 (Yugoslav Acad. Sci. Arts, Zagreb, 1978).

9. Institute of Medicine, National Academy of Sciences Tem perature Stable Vaccines for Developing Countries: Signifi-
} cance and Development of Strategies (Washington, DC, 1987). filaments from myosin.
At a recent meeting ${ }^{y}$, it was pointed out that consistent use of stabilizers such as $\mathrm{MgCl}_{2}$ would help minimize reliance on the cold chain. Such stabilized vaccine, although not perfect, would meet the suggested target of stability for 3 days at $37^{\circ} \mathrm{C}$ with a maximum loss in potency of $0.5 \mathrm{log}$.

Department of Virology and JOSEPH L. MELNiCK

Epidemiology,

Baylor College of Medicine,

Houston,

Texas 77030 ,

USA

\section{Myosin heads in muscle thick filament assembly}

SIR-Morel and Bachouchi' discuss the role of myosin heads in the assembly of skeletal muscle thick filaments. They cite evidence to show that the binding of MgATP to myosin heads is crucial in the assembly of thick filaments of physiological diameter. Although data in support of these head-mediated phenomena appear reasonable, they may be irrelevant to the generation of native-like

Synthetic myosin filaments assume different morphological forms depending on the nature of the filamentogenic solvent. The experiments cited by Morel and Bachouchi were carried out on what are conventionally called the $p \mathrm{H} 7$ class of filaments. These differ from in vivo filaments in that they are generally spindleshaped, thicker and lack a clearly demarcated bare zone at their centres ${ }^{2.3}$.

As pointed out', the addition of MgATP to the $p \mathrm{H} 7$ class of filaments does appear to make them more native-like in structure. On the other hand, this treatment seems to be unnecessary with filaments of the $p \mathrm{H} 8$ class. These filaments are morphologically similar to native filaments save that they are shorter and have a narrow length-distribution rather than the precise length seen in vivo ${ }^{2.3}$. Interactions between the myosin heads and the filament core seem to be minimal, as the heads and part of the tail (the S-2 segment) are predominantly dissociated free of the filament surfaces ${ }^{4.5}$. This observation effectively excludes head-core interactions from playing a significant role in their assembly.

The limit on length can be overcome by copolymerizing the myosin with C-protein ${ }^{6}$, a constituent of the native filament. The longer filaments so formed retain the physiological diameters typical of $\mathrm{pH} 8$ and native filaments. The only gross structural property to distinguish them from native thick filaments is the lack of a precise length. A crucial role for head-head and head-core interactions and/or for MgADP or MgATP in assembly therefore seems unlikely. It is more plausible that the head-related phenomena function in contraction than in assembly (see ref. 7).

Julien S. Davis

Department of Biology,

The Johns Hopkins University,

34th and Charles Streets,

Baltimore,

Maryland 21218, USA

1. Morel. J. E. \& Bachouchi, N. Nature 332. 591 (1988).

2. Kaminer. B. \& Bell. A. L. J. molec. Biol. 20, 391-401 (1966).

Josephs. R. \& Harrington, W. F. Biochemistry 5, 3474 3487 (1966).

4. Sutoh. K., Chiao, Y.-C. C. \& Harrington, W. F. Biochemistry 17, 1234-1239 (1978).

Reisler, E. \& Liu. J. J. molec. Biol. 157. 659-669(1982)

6. Davis. J. S. J. Muscle Res. Cell Motil. (in the press).

7. Davis, J. S. A. Rev. Biophys, biophys. Chem. 17, 217-239 (1988)

\section{Designing antibodies for human therapies}

SIR-The recent descriptions ${ }^{1.2}$ of reshaping of human antibodies represent remarkable feats of genetic engineering with potential to produce reagents useful in human therapy. It is anticipated that these would be superior to rodent antibodies as immunotherapeutic agents because treated individuals should not develop antibodies to the human variableregion framework and constant regions of the heavy $(\mathrm{H})$ and light $(\mathrm{L})$ chains of immunoglobulin molecules. The concern remains, as explicity expressed by Riechmann et al. ', that the idiotypic portion of the reshaped human IgG1 antibody that they hope to use in therapy may be immunogenic.

In that regard, I wish to raise the issue of $\mathrm{H}^{-}$and $\mathrm{L}$-chain $\mathrm{Gm}$ and $\mathrm{Km}$ allotypes. Although antibody responses to the $\mathrm{Km}$ and $\mathrm{Gm}$ allotypes may not themselves present major problems, the presence of foreign allotypes may provide carrier determinants and thus enhance immune reponses to idiotypes. In inbred strains of mice, immunization with myeloma proteins bearing allotypes foreign to the recipient strain elicits anti-idiotype more readily than when syngenic strains are immunized ${ }^{5}$.

I therefore suggest that those planning clinical studies consider $\mathrm{Km}$ and $\mathrm{Gm}$ matching their recipients with the therapeutic antibodies. Both the reported human IgG1 clones $^{3,4}$ have sequences corresponding to the $\mathrm{Gm} 1$ type in $\mathrm{CH} 3$ (Arg-Asp-Glu-Leu at 355-358) and to Gm17 (Lys 214) in CH1. Because of the success of site-directed mutagenesis experiments $^{1.2}$ it should be simple to introduce the necessary point mutations to produce reagents with the alternative allotypic forms of human IgG1 and $\kappa$-light chains. It will be considerable interest and benefit if allotype-matching prolongs the time period of effective 